\title{
AN INVESTIGATION OF SOCIABILITY MEASUREMENTS IN PROXIMATE ONLINE COMMUNITIES
}

\author{
Nelson Wright, Alison Varey \& Thomas Chesney \\ School of Computing \\ Napier University \\ Edinburgh
}

\begin{abstract}
This paper reports the results of an investigation into measurements of the sociability of proximate online communities, in other words, how well it supports social interaction rather than in terms of its usability, which tends to focus on task issues and user satisfaction. Measurements that have been proposed by other writers are investigated and several new measurements are proposed. These were tested during the introduction of a new proximate online student community called Veritas. The results showed that sociability is best measured by a combination of qualitative and quantitative approaches.
\end{abstract}

\section{KEYWORDS}

Sociability, online communities

\section{INTRODUCTION}

Preece (2001) defines an online community as '[a]ny virtual social space where people come together to get and give information or support, to learn or to find company. The community can be local, national, international, small or large'. Hung and Chen (2002) take a different perspective, and argue that online communities can be categorised as either a community or a quasi-community. They define a quasicommunity as one where users interact because 'there is a rather quick way of receiving some (personal) benefit, such as having some query answered by someone, somewhere in the Internet world' (Hung \& Chen, 2002, p26). In contrast, they define a true online community as one where participants learn to take on an identity (for example, a doctor, in a community of practice), as well as having the knowledge needed to do a task. This idea of having an identity in cyberspace is a common theme to many online communities, and can be represented by pseudonyms, graphical depictions, indication of gender, small profiles of the user and avatars. Rheingold (1994), writing about his experience of one of the first online communities, the San Francisco WELL (Whole Earth 'Lectronic Link), states, '[n]o single metaphor completely conveys the nature of cyberspace. Virtual communities are places where people meet, and they are also tools; the place-like aspects and tool-like aspects only partially overlap.' This aspect of doing an activity is what makes online communities stand apart from merely passively looking at web pages. Obviously, members of an online community look at web pages too, but the users often come to participate in some manner, whether that is to contribute to a discussion, upload a music file, add a URL to a list of resource links, or rate other users' contributions. The definition of online community used here is 'a virtual meeting place where people share thoughts, ideas, and resources'.

One possible way of defining online communities is in terms of whether it serves a geographically proximate community, or a more dispersed community. The proximate online community presents a special case, as there will be opportunities for the members to communicate face-to-face in reality, as well as online in virtual reality. This could have repercussions, such as whether members would make the effort to interact online if they thought they might see a person later that day or week. Also, it might moderate the behaviour of some members, for example they might be less aggressive in their message posts if there was a chance they might meet the person face-to-face. There is a high probability that proximate online communities will 
also have been in existence prior to the creation of the online community, and so the participants will already have formed some ideas about the nature of the community and the participants, and the roles some members may play within that community. As Wellman and Gulia (1999) state, 'people do not neatly divide their worlds into two discrete sets: people seen in person and people contacted online. Rather, many community ties connect offline as well as online. It is the relationship that is the important thing, and not the communication medium.' Additionally, there will be a shared history and culture amongst the majority of participants.

This paper reports the results of an investigation into measurements of the sociability of proximate online communities. It looks at how well they support social interaction rather than in terms of their usability, which tends to focus on task issues and user satisfaction. Measurements that have been proposed by other writers are investigated and several new measurements are proposed. These were tested during the introduction of a new proximate online student community called Veritas.

The rest of the paper is organised as follows. Section 2 briefly examines the concept of sociability and the determinants for measuring it. Section 3 describes a pilot study undertaken to establish the sociability measurements to be used for Veritas, the online student magazine, explaining where they came from, how they are to be measured and why they were used. Section 4 describes the results of the applying these measurements to Veritas and section 5 concludes with the strengths and weaknesses of the measurements.

\section{SOCIABILITY}

Preece (2001) examined the concept of sociability for online communities, meaning the ease with which members can interact and communicate with each other. She identified three determinants for sociability: purpose, people and policy. For purpose, she identified such criteria as number of messages sent, how ontopic the discussion is, and how much interaction is taking place. For people, she identified such criteria as how many and what kind of members a community has, and the roles the members play. For policy, she identified how effective any moderating policies are to control unwanted behaviour, any policies to encourage the development of relationships, and the level of trust.

An analysis of Usenet newsgroups by Smith (1999, p.204) revealed that one way of measuring the quality of interaction on a bulletin board was by measuring the 'poster-to-post' ratio (i.e. the total number of members who post messages divided by the total number of posts). Smith found that a ratio close to one indicated a 'lack of turn taking', as each member writes a message only once, and no one responds to it. Turn-taking is indicative of direct social interaction (Sacks et al. 1974, cited by Smith 1999, p.204). This could be perfectly reasonable however in a bulletin board primarily set aside for announcements, or for imparting information without the need for discussion. A low ratio however, shows that a few people contribute most of the postings. This could be due to a lack of interest in the site/topic, or it could be due to the prevalence of members who dominate the discussions, and this could scare people away. A 'medium' ratio between zero and one was found by Smith to be more indicative of interaction between members, although he stressed that it did not always guarantee that people actually responded to the messages left by other members.

One issue that communities face is that of lurking, that is, people read messages but do not contribute their own messages. However, because it is not easily observed online, it is less detrimental to group morale than in similar face-to-face situations (Wellman and Gulia, 1999, p. 180). In fact, lurkers may provide a useful role in that many message boards count the number of times a message has been viewed and display this information. So someone who posts a message may notice that many people have read their message, and feel satisfied that their views are being noted, even if there are few or no replies.

Girgensohn \& Lee (2002) have approached matters from a different perspective, using the idea of 'social interaction components' to support sociability. These components are: 1) common ground, 2) awareness, 3) interaction enablers and mechanisms, 4) place-making. Common ground is the shared understanding between participants that allows communication to occur reasonably hitch-free. Examples include the availability of member's profile data, and the seeding of content in an area of interest to all the participants. Awareness is the way in which users can understand and interpret other users' actions in the context of the online community. This can be in the form of showing what new postings have occurred since the member's last visit. It might also show what upcoming events might be taking place. The interaction enablers are the means 
by which members know what the availability and type of social interaction choices are. These are typically GUI components and hyperlinks. The mechanisms themselves can range from quick polls, the forums themselves, to ways of sending another member a private message. Place-making is when the members create a 'sense of place' by adapting the social and cultural norms to suit themselves. For example, when the discussion boards are used not as just a means for information sharing, but as a way of co-ordinating social meetings, and for formulating a set of informal 'rules' or norms for using the site.

Rules governing group behaviour are an important aspect of sociability, and Ostrom (1990) has formulated a set of rules that appear to govern successful real-life communities. Amongst these is the ability for members to customise the rules that govern their behaviour. This is echoed by Kim's research about members being 'empowered over time' (Kim, 2000), and shows that these rules are applicable to virtual communities too. Both Ostrom (1990) and Godwin (1994) also found that the most effective way of resolving disagreements within a community is by the members themselves. Another important design principle of Ostrom's is that group boundaries should be clearly defined. This in theory should make it clear who can use collective resources and it should prevent people from entering the community and taking from it without contributing themselves. This has been achieved online in part at least by requiring users to register before making use of certain features of the online community.

\section{PROPOSED MEASUREMENTS}

An online community was analysed for sociability using the criteria given in section 2 to investigate the suitability of, and how easy it was to obtain, each measurement. The results of this analysis are now discussed.

The analysis used Preece's sociability determinants; purpose, people and policy as a starting point, to see how effective they were. Many of the measurements were easy, if time-consuming, to obtain. However, problems were found with Preece's people determinant: missing information, such as age and gender of the participants, with only a small minority of the members volunteering this information. Other problems encountered were the qualitative nature of purpose criteria. For example, at what point does a message become 'off-topic' and how can rating reciprocity be achieved? Preece herself is vague on the last question, and suggests in some way measuring the 'ratio of giving and taking' from a community (Preece, J. 2001, P 351). This would involve investigating how many questions a member asked, compared to how many responses they gave. Again, this could prove problematic, for example, there might be a debate over whether a question was asked rhetorically. Any purely quantitative approach would take little account of the meaning and content of each message, something that is surely less than desirable when analysing an online community, where language is usually the common currency.

Additional purpose criteria were therefore added to classify messages. The classifications are: opinion; agreement; disagreement; facts and information; and maintenance. These help to give an answer to Preece's sociability questions of 'How much and what kind of reciprocity occurs?'. 'Opinions' is a straightforward classification when a post is stating a member's views on an issue. 'Agreement' and 'disagreement' is in relation to a previous post. The 'facts and information' category could be viewed as a measure of how much a member is giving to the community, in the form of information or the role that the member is taking on. The 'maintenance' category indicates any posts that support the upkeep and running of the community, such as members saying that they would organise a night out, or find out some information which would benefit the whole community. The ratio of the category in each classification should reflect the nature of the social interaction taking place.

A 'responsiveness index' was also developed that investigated the number of new topics created per day. By dividing the number of posts per day by the number of topics per day, an estimate could be made of the interactivity occurring in the community, a 'responsiveness' index. If it had a value of 1 or close to 1 , then it could be assumed that the board was used mainly for announcements, and little social interaction was taking place. If the responsiveness index had a higher value, it would indicate that at least members were replying to each other's posts, although whether this was friendly in nature would have to be determined by a message analysis.

It proved difficult to assign a value to the amount of lurking going on, without detailed web logs. However, an estimate can be found by comparing the number of views a message has to the number of posts. 
If it assumed that a person who posts a message, reads that message once, to check for errors, then dividing the number of views of a message by the number of posts should give a measure of the practice of lurking, if not the exact number of lurkers. This is obviously an approximate measure.

Smith's quantitative measure of poster-to-post ratio and Girgensohn \& Lee's four qualitative determinants of social interaction components (2002) were also successfully applied.

To summarise, the measurements of sociability that were used in the study were the quantitative measures of: number of registered members, number of messages, posts per day, topics per day, thread depth and breadth, poster-to-post ratio, lurker index and responsiveness index. Message classification was chosen to categorise the posts into opinions, agreements; disagreements; facts and information and maintenance. The qualitative determinants of common ground, awareness, interaction enablers; place-making; trust; recognition of contributions and guest posting were also used.

\section{RESULTS}

What follows are the results of the assessment of the sociability determinants and measures through the development of a proximate online community as part of a student newspaper's website, Veritas Online.

\subsection{Quantitative Measurements}

Between the start date of $6^{\text {th }}$ February 2003 and $24^{\text {th }}$ April 2003, 30 members registered and 804 messages were posted. The administrator posted 30 of the messages in order to seed the community and also to encourage posting by offering a competition by which any poster could win a pair of cinema tickets. By $24^{\text {th }}$ April, 804 posts had been made in 45 topics, giving an average of 10.37 posts per day and 0.57 topics per day. Preece (2001, p.351) cautions against using this as a measure of sociability by itself, as it does not take into account 'quality of the social interactions'. However, when used with other sociability determinants, it is certainly a useful measure of how much activity takes place within the online community. Also, if the number of posts per day is very low, it has to be assumed that most people are not posting messages, and the sociability of the online community could legitimately be questioned.

Table 1. Quantitative measurements for Veritas Online message board

\begin{tabular}{ll}
\hline Measurement & Value \\
\hline Number of registered members & 30 (1 of whom was the administrator) \\
Number of messages & 804 (30 due to the administrator) \\
Posts per day & 10.37 \\
Topics per day & 0.57 \\
Thread depth & 17.9 \\
Thread breadth & 47 \\
Poster-to-post ratio & 0.02 \\
Lurker index & $7.39 *$ \\
Responsiveness index & 18.19 \\
\hline \multicolumn{2}{c}{ *Average taken from a sample of forums analysed }
\end{tabular}

Thread depth was long at 17.9, which indicated a high degree of reciprocity and social turn taking (Smith 1999). This was backed up by the fact that thread breadth was only 47, indicating a deep and narrow thread architecture, which meant that members tended to respond to existing posts rather than creating new topics. Preece suggests that this may be the case in 'scholarly discussion communities' (Preece, J., 2001, p. 351), which tend to have a less empathic community. This was not the case for the Veritas Online community however, as members often empathised with each other, and 'scholarly discussions' were rare. Indeed, one of the few unanswered posts was one making a serious point about the anti-war stance of many student bodies.

Smith's poster-to post ratio was very low, being 0.02 , which indicated that a small number of people were making a lot of posts (Smith 1999), which was indeed the case.

In terms of 'lurking', the number of posts, views and the 'lurker index' is given in Table 2 below. They are split into three of the forums Veritas was organised into, messages about courses, messages about recreation, and wish list. 
Table 2. Lurker indicators for Veritas Online message board

\begin{tabular}{lrrc}
\hline Forum & Posts & Views & Lurker Index \\
\hline Courses & 104 & 930 & 8.94 \\
Recreation & 79 & 538 & 6.81 \\
Wish List & 147 & 942 & 6.41 \\
\hline
\end{tabular}

As can be seen, the amount of lurking is fairly similar between the 'Recreation' and 'Wish List' boards, despite the disparity in the number of messages posted. An explanation for the higher lurker index on the courses board could be that members are more interested in reading about information that other members have contributed to this board, as this may benefit them. This seems to be backed up by the message classification analysis (see section 4.2), which shows that $36 \%$ of the posts for the courses board fall in to the 'facts and information' category, but only $27 \%$ and $22 \%$ fall into this category for the wish list and recreation boards respectively, which are more opinion-based.

The responsiveness index is 18.19, which shows that there is considerable social interaction going on, and members were replying to each other's posts.

\subsection{Message Classification}

In the case of Veritas Online, the board contained 804 messages by $24^{\text {th }}$ April 2003, too many to contemplate a detailed qualitative analysis of every message. Therefore, a sample was taken from the three forums, courses, recreation and wish list. The classification of messages across the forums were as follows: opinions $39.6 \%$; agreements 31.6\%; disagreements 5\%; facts and information $21.1 \%$; and maintenance $2.7 \%$. A more detailed breakdown by forum is given in figure 1 .

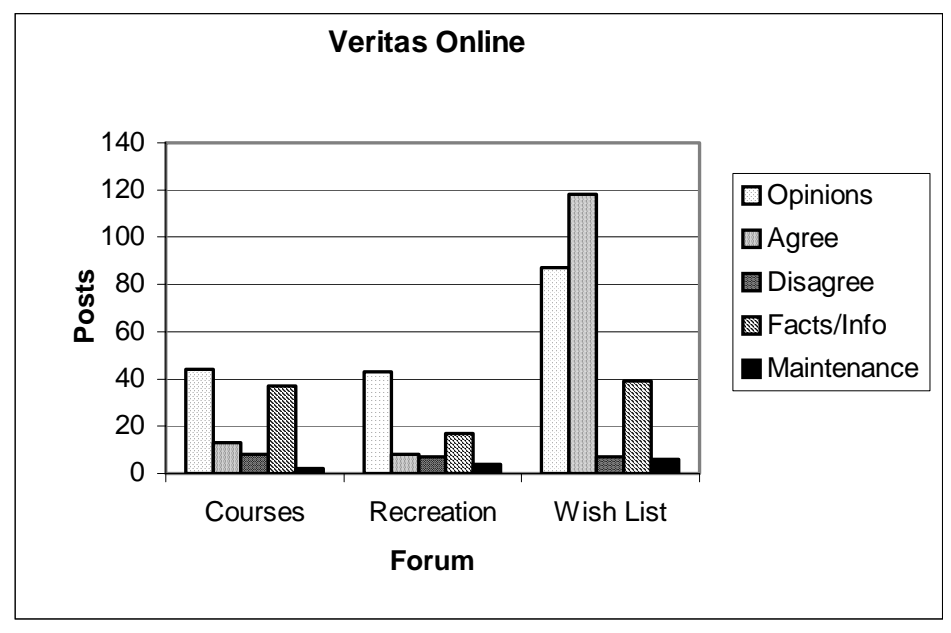

Figure 1. Message classification of the Veritas Online message board

An interesting aspect noted while compiling the statistics was that almost none of the initial thread topics bore any relation to what was being discussed in the most recent postings on that thread. Preece (2001, p.351) suggests using a measure of how 'on-topic' are the threads as a method in determining sociability. However, this does not take into account the possibility that a perfectly valid, sociable discussion could have evolved over time to become 'off-topic'. Determining that an online community is less sociable as a result of these threads appears to be too crude a measure. Closer moderation of the board could have solved this difficulty, but it was felt that this would discourage members from posting, and confuse the current members if the posts were moved.

The 'Wish List' contains the highest percentage of agreement, this was caused by members agreeing to each others proposals. It was also interesting to note that as the forum with the most posts, 'Wish List' did not have any more disagreements than the other two boards. This indicates that in this context disagreements do not have a direct influence on generating posts. At times they appeared to spark a reply, and at other times they seemed to 'kill off' a thread, so their effect was cancelled. In the case of the 'Wish List' forum, the reverse was the case; agreement increased sociability and encouraged more posting of messages. 


\subsection{Qualitative Determinants}

Common ground between members was demonstrated by their reference to artefacts and shared experience in the proximate community, such as issues about the toilets and examinations. Awareness of other members of the community was achieved by the profiles of the members being publicly viewable and members being able to see who else is using the message board at any time. Members can also see new posts since their last visit and can view unanswered posts, which encourages replies and fosters sociability. The interaction enablers employed were: forums for specific topics, large buttons for posting and replying to messages, options to start polls or reply to polls, private messages could be sent to members, threads could be displayed chronologically or sorted and hyperlinks could be included in messages encouraging sharing of information and resources. Place-making was achieved by allowing members to change the look of the board and create their own topics and polls.

Trust was fostered by members having a registration agreement to sign up to, and email addresses are displayed in a member's profile. Also, the IP address on all posts were logged.

The community had a system whereby members were given a rank according to how many posts they had put on the board and this seemed to be popular amongst the members. There were often posts devoted to that topic alone, and comments were made when members achieved a new rank. According to the messages posted, this apparently trivial ranking scheme did appear to be a major motivational factor in encouraging members to post, and appeared to be the main factor in raising the posts per day from 5.24 to 10.37. This backs up research indicating that schemes that show status within the community encourage participation. The ranks were decided automatically with the board administrator setting the number of posts required for each rank. The more fanciful rank titles seemed to work best with the students on the Veritas board, such as 'Message Board God', and 'Pure Energy Being'. With a ranking system, members can only increase their status by giving to the community in the form of posting messages (Kelly et al 2002).

Guest posting is possible, the user can choose whether or not to use their real name, or pseudonym, or to leave this field blank.

\section{CONCLUSION}

Each of the measurements that were investigated is now discussed. The number of registered members, number of messages, number of posts per day and number of topics per day all give useful information on social activity, but do not give the whole picture of how sociable an online community is. These measures need to be combined with other measurements to give more detailed information about the quality of the social interaction. Using Smith's poster-to-post ratio (1999) partially overcomes this problem, and combining that with the lurker index and responsiveness index, one can start to build up a better picture of the sociability of the online community.

The thread depth and breadth does give a measure of the type of social interaction, with a narrow and deep architecture suggesting a more discussion based community and a broad and shallow architecture suggesting more announcement rather than discussions. However, this measure can be affected by the fact that responses to a posting are not always "on topic", and a deep and narrow architecture does not definitely show a detailed discussion on one topic, but can represent a wide-ranging discussion. Again this measure would best be combined with other measures such as how "on topic" a message is, although as was stated before, this is not easy to quantify. Likewise, the responsiveness index estimates the interactivity occurring in a community. However this measure, like the thread depth, is affected by people responding to a posting with a completely unrelated message. A more detailed message analysis would be required to measure how truly responsive a community is.

The poster-to-post ratio is intended to show the kind of social interaction, with a low ratio characterising a small number of core people who contribute most of the postings. However this is a crude measure that can be affected by one member making a large number of postings. Furthermore, a high poster-to-post ratio could represent either members' lack of interest in the topics or a fear of contributing. Only by asking the members, can the reasons for non-contribution be discovered.

The lurker index gives an indication of the amount of lurking behaviour for messages but cannot be seen as an accurate measure of the number of lurkers. Lurkers could be defined as people who return at least once 
to the message board but do not post any messages. In order to measure this, detailed logging of users is required.

Message classification was chosen to categorise the posts into opinions, agreements; disagreements; facts and information and maintenance. However, these classifications are not exclusive or exhaustive and a message can combine, for example, agreement with factual information. A method needs to be found of classifying such messages. Since the classification is also quite subjective, it would be interesting to arrange for at least two people to classify the messages to see the validity of their classifications. The message classification helps to explain why certain forums had differing levels of posts, lurking and responsiveness. Moreover a way of categorising messages into more than four categories might be desirable in future. For example, a category used when members refer to shared artefacts in the proximate community could be used.

The more qualitative determinants proved interesting to apply, such as whether place-making was actually taking place, and in what way awareness was enabled (Girgensohn. \& Lee, 2002). A more formalised system of investigating this aspect could be introduced, such as examining the profiles of members to see how much information they had provided. Measuring trust is important but this is difficult to achieve so policies were put in place to encourage trust. In future, a method of recording how many times a member's identity, or assertions made, were questioned could be a method of measuring trust in the community. Common ground is a useful measure in a proximate online community, as it captures shared experiences that are perhaps absent from non-geographic communities. Moreover, it was clear from the case study, that making members of the community aware of others through members' profiles and additional data (such as who is using the message board at any time and unanswered posts) can foster sociability. Similarly, interaction enablers can encourage sociability through sharing of information and resources but it is hard to measure the extent to which they do. Place-making did not seem to be as important in fostering sociability.

The fact that non-members can post messages can increase sociability, as requiring members to register to engage with the community could act as a bar to participation. A measurement of this phenomenon could be useful in the future.

Measuring sociability in online communities is still in its infancy, and the various measures presented here are not envisaged to be the final solution for all online communities. An approach that combines different techniques and measures, adapting these to suit the needs of measuring the sociability of the virtual community in question, needs to be sought. Applying some existing determinants for sociability and creating new measures gave an insight into how useful these tools are for practical use.

\section{REFERENCES}

Girgensohn, A. \& Lee, A. (2002). Making web sites be places for social interaction. CSCW '02 November 16-20, 136145.

Godwin, M. (1994). Nine principles for making virtual communities work. Wired 2.06 (June), 72-73.

Hung, D. \& Chen D (2002). Understanding how thriving internet quasi-communities work: Distinguishing between learning about and learning to be. Educational Technology, Jan-Feb 2002, 23-27.

Kelly, S. U., Sung, S. \& Farnham, S. (2002) Designing for improved social responsibility, user participation and content in on-line communities. Human Factors In Computing Systems, CHI 2002 Conference proceedings, ACM, New York, April 20-25, 391-398.

Kim, A. J. (2000). Community Building on the Web. Peachpit Press, Berkeley, CA.

Kollock, P. (1996) Design principles for online communities. Harvard Conference on the Internet and Society, 1996.

Ostrom, E. (1990). Governing the Commons: The Evolution of Institutions for Collective Action. New York: Cambridge University Press.

Preece, J. (2001). Sociability and usability in online communities: determining and measuring success. Behaviour \& Information Technology, 20(5), 347-356.

Rheingold, H. (1994). The Virtual Community, London, Martin Secker \& Warburg.

Smith, M. A (Eds) (1999). Invisible crowds in cyberspace. In Smith, M. A. \& Kollock, P. (Eds) Communities in Cyberspace London: Routledge.

Wellman B, \& Gulia, J. (1999). Virtual Communities as Communities: Net surfers don't ride alone. In Smith, M. A. \& Kollock, P. (Eds) Communities in Cyberspace London: Routledge. 